\title{
Multivariate simulation with DATASIM: The Mihal and Barrett study
}

\author{
DRAKE R. BRADLEY \\ Bates College, Lewiston, Maine
}

\begin{abstract}
A general-purpose data simulator, DATASIM, allows instructors and students to easily generate simulated data for experimental, crossbreak (frequency table), and multivariate research designs. Students can select any of a variety of data analysis problems from the built-in library and then obtain problem descriptions and "individualized" data sets to analyze and interpret. DATASIM can also be used to generate, plot, and analyze interesting data sets for discussion in class, to demonstrate principles of sampling theory and research design, and to conduct sampling experiments (Monte Carlo research). Finally, the electronic blackboard capability of the program allows instructors to conveniently display multiple sources of information, such as outlines, graphs, sample data sets, ANOVAs, and the like. The present paper focuses on the techniques and methods required to perform multivariate simulation. To this end, DATASIM is used to simulate a study by Mihal and Barrett (1976), which predicted auto accidents from measures of perceptual style, reaction time, and selective attention.
\end{abstract}

This paper describes a software package called DATASIM. ${ }^{1}$ The program was specifically designed to generate simulated data for studies in the social and behavioral sciences (Bradley, 1988, 1991b). The simulations can be based on actual studies published in the literature, or on hypothetical studies developed to demonstrate particular principles of experimental design, research methodology, data analysis, or sampling theory. The software is supplied with a library of built-in problems that instructors can use for in-class demonstrations and that students can use to obtain simulated data for homework assignments. Moreover, by saving problem description and initialization files in appropriate subdirectories of the library, instructors can easily augment the library with problems of their own devising (Bradley, 1988, pp. 88-94).

For any given problem, DATASIM generates independently randomized data sets for each student to analyze and interpret. Since each data set is associated with a specific seed used to initialize the random number generator, it is a simple matter to have the program generate

DATASIM was developed by the author and is available at nominal cost from Desktop Press, 90 Bardwell St., Lewiston, ME 04240 (telephone: 207-786-4113 or 6180). The computer simulations reported in this paper were conducted on a Macintosh IIci supplied by Apple Computer and the Consortium of Liberal Arts Colleges. The author is indebted to Apple and the CLAC Grant Review Committee for an Equipment Grant which supported the present research and, more generally, the development of DATASIM for the Macintosh. The author also wishes to acknowledge the support of NSF-ILI Grant USE-8852194, awarded to Bates College by the National Science Foundation (G. Nigro and D. R. Bradley, principal investigators). The grant funded the creation of a microcomputer laboratory used by students to conduct DATASIM simulations and homework assignments. Requests for reprints should be addressed to D. R. Bradley, Department of Psychology, Lewiston, ME 04240. the correct solutions ( $t$ tests, ANOVAs, multiple regression analyses, etc.) for a particular data set. This strategy has two major advantages. First, since each student's solutions are unique, he/she must carefully check the results for reasonableness. This encourages the student to develop important error-checking skills. Second, the variation in the pattern of results obtained across students will accurately reflect the inherent sampling error expected when one is conducting independent replications of the original study. The fact that students get somewhat different results, and may even come to opposing decisions with respect to $H_{0}$, has interesting pedagogical ramifications. The astute instructor will see this as an opportunity to develop the concepts of sampling error, statistical power, Type I and Type II decision errors, and the like (Bradley, 1991b, p. 18).

In comparison with the traditional approach of having students flip coins, roll dice, or draw numbers from a hat, DATASIM provides highly flexible methods for generating data. The program allows the user to generate data from discrete or continuous probability distributions, from normal or nonnormal distributions, and from populations having correlated or uncorrelated observations. Furthermore, intact data sets can be generated for a variety of research designs. For example, data can be generated for one-way experimental designs having independent groups, matched groups, or repeated measures across conditions, and for two-way or three-way factorial designs having any combination of independent and correlated groups factors. As shown below, multivariate data sets can be generated as well, with whatever intercorrelation or multiple regression structure is desired by the user. Finally, frequency data can be generated for either one-way or two-way tables, and in the latter case, the row or column marginal fre- 
quencies can be either fixed or random. Once generated, the data sets can be plotted and analyzed by DATASIM. ${ }^{2}$

A unique feature of the program is the ability to perform repetitive simulation - that is, to generate and analyze multiple simulated data sets, save the results in a file, and process the resulting sampling distributions. This capability allows the user to conduct sampling experiments to investigate the effects of violating assumptions (e.g., normality, homogeneity of variance, sphericity, homoscedasticity, minimum expected frequency, etc.) on the Type I and Type II error rates of various statistical tests. Previous publications have described the basic design and capabilities of the software (Bradley, 1989a, 1989b), evaluated the accuracy of the random number algorithms (Bradley, Senko, \& Stewart, 1990), reviewed the methodology for conducting a simulation of a previously published field experiment (Bradley, 1991a), and illustrated a number of laboratory simulations useful for teaching principles of sampling theory and inferential statistics (Bradley, Hemstreet, \& Ziegenhagen, 1992). The present paper reviews the techniques for conducting a simulation of a multivariate study.

\section{The Mihal and Barrett Study}

In 1976, Mihal and Barrett published an interesting study in the Journal of Applied Psychology, in which they examined the relationship between measures of perceptual style, reaction time (RT), selective attention, and the number of automobile accidents experienced by drivers. They hypothesized that individuals who were field dependent, slow in making decisions, and poor at maintaining selective attention would have more accidents. They used the rod-and-frame test (RFT) and the embedded-figures test (EFT) to measure perceptual style. These tests are designed to assess the individual's ability to extract salient information from a complex background (Witkin, Dyk, Faterson, Goodenough, \& Karp, 1962). In the RFT, the observer is required to adjust a luminous rod (in an otherwise dark room) to match the gravitational vertical. The rod is surrounded by a tilted luminous frame. In the EFT, the observer is required to find and trace out a form embedded in a more complex figure. Individuals who are field dependent do not perform well on these tests: they are more likely to align the rod with the tilted frame rather than the true vertical, and they take longer to identify the embedded figures. Field-independent observers, on the other hand, show only small deviations (in degrees) of the rod from vertical, and detect the embedded figures fairly quickly. Mihal and Barrett reasoned that individuals who had difficulty in ignoring irrelevant context (the frame in the RFT) or who had difficulty in disembedding stimuli from a confusing background (the embedded figures) would be involved in more automobile accidents.

Four measures of RT were obtained by testing subjects in an automobile simulator. The initial and simple RT measures consisted of the time taken to initiate and complete, respectively, a simple motor task (e.g., depressing the brake pedal). For the choice RT measure, the time taken to complete the appropriate motor response for one of three visual stimuli (a green left-turn arrow, a red braking disk, or a green right-turn arrow) was recorded. Finally, in the complex RT task, the subject was presented with a photograph of an actual driving scene in which was embedded a signal or sign directing a particular action (turn left, stop, turn right). The time required to complete the required action was used as a measure of complex RT. Mihal and Barrett (1976) predicted that the measures of choice and complex RT would predict accident rates, whereas measures of initial and simple RT would not. This prediction was based on the assumption that the RT task must involve some element of decision making, analogous to those encountered in actual driving situations, in order for it to reliably predict accident rates.

Finally, a measure of selective attention was obtained by having subjects monitor dichotic messages simultaneously presented to the two ears. A tone indicated which of the two ears was relevant at any given point in time, and the subject's task was to repeat as many of the target items (digits) as possible from the set presented to the relevant ear. Since the subject's score consisted of the number of errors made, high scores on this measure indicated poor selective attention. Mihal and Barrett (1976) predicted that subjects with poor selective attention would be involved in more accidents.

To test these various hypotheses, $N=75$ drivers of utility company vehicles were tested on the perceptual style, RT, and selective attention measures. The number of accidents each driver experienced over the preceding 5 years was determined by accessing company records. The seven predictor variables were then intercorrelated with each other and with the criterion variable (accidents). As predicted, field dependence on the RFT and EFT was positively correlated with accidents $(r=.38, p<.001$, and $r=.24, p<.05$, respectively), and drivers with poor selective attention tended to have more accidents $(r=.40$, $p<.001)$. Complex RT was the only RT measure that correlated significantly with accidents $(r=.27, p<.01)$. Given that Mihal and Barrett (1976) were clearly interested in predicting accident rates from the measures of perceptual style, RT, and selective attention, it is somewhat surprising that no multiple linear regression analyses were reported. This point is explored in greater detail below.

The Mihal and Barrett (1976) study provides an excellent example of the use of multiple correlation and prediction in applied research. The theoretical issues are relatively easy for students to grasp, and the topic of the studythe relationship between "distractibility," decision-making time, and susceptibility to car accidents-is inherently interesting. Furthermore, a number of statistical issues are raised by the study. For example, while it is true that the perceptual style, complex RT, and selective attention measures predict accident rates, the $r^{2}$ values for these predictors are relatively small, ranging from .06 to .16 . So there is quite a bit of unexplained variance to be accounted for by other, as yet unknown, factors. Also, by comput- 
ing a multiple linear regression analysis and comparing the value of $R^{2}$ to the individual values of $r^{2}$, the instructor can show how the predictors account for partially overlapping and redundant sources of variability in the criterion measure. ${ }^{3}$ Finally, as we will see below, the distributions of the predictor variables are clearly nonnormal, the criterion variable is both nonnormal and discrete, and there is a fairly good chance that the homoscedasticity assumption is not tenable. Is it reasonable to conduct a multiple linear regression analysis on such data, and can the results of the analysis be trusted? DATASIM sampling experiments based on the Mihal and Barrett study can provide preliminary answers to these questions.

\section{Determining the Simulation Parameters \\ for Mihal and Barrett}

To simulate a multivariate study by using DATASIM, we need to specify the mean, standard deviation, and distribution shape for each variable, the intercorrelations among the variables, and the overall sample size $(N) .{ }^{4}$ The sample statistics reported in the original study may be taken as estimates of the corresponding population parameters, and histograms of the individual distributions (if available) may be used to estimate the skew and kurtosis for each variable. Table 1 presents the means and standard deviations reported by Mihal and Barrett (1976) for the seven predictor variables. Unfortunately, the mean and standard deviation of the criterion variable (accidents) were not reported. However, it is possible to estimate these values from information provided in Table 1 of Mihal and Barrett's report (1976, p. 231): a note accompanying the table indicates that of the $N=75$ drivers in the study, $n=29$ had no accidents, $n=15$ had one accident, and $n=31$ had two or more accidents. If we make the assumption that all of the drivers in the "two or more" accident group had exactly two accidents each, then the mean and standard deviation for this measure would be 1.03 and .90 , respectively. Of course, these values will underestimate the true values to some extent, because an unknown number of drivers actually had three, four, or more accidents. However, in the absence of precise information about the actual distribution of the accidents, these estimates will suffice to illustrate DATASIM's ability to simulate multivariate research studies.

Given that each of the variables in Table 1 has a lower scale limit of $X=0$, the means and standard deviations suggest that several of the variables were nonnormally distributed. The RFT measure, for example, has a mean of 4.04 and a standard deviation of 3.36. Since the mini- mum score for the RFT is zero, this indicates that the data for this variable are positively skewed. ${ }^{5}$ The same argument applies to the EFT, selective attention, and accident measures. Furthermore, although the RT measures do not have means that are within 3 standard deviations of the lower scale limit, it is quite likely that these data are positively skewed as well. Therefore, to properly simulate Mihal and Barrett's (1976) study, we will need to specify appropriate nonnormal distributions for each of the variables.

DATASIM uses the generalized lambda distribution (GLD) to approximate a wide variety of unimodal symmetric and asymmetric distributions (Mykytka, 1978; Ramberg, Dudewicz, Tadikamalla, \& Mykytka, 1979; Ramberg \& Schmeiser, 1972, 1974). The GLD is a fourparameter percentile function defined as follows (Ramberg et al., 1979, p. 202):

$$
R(p)=\lambda_{1}+\frac{p^{\lambda_{3}}-(1-p)^{\lambda_{4}}}{\lambda_{2}} \quad(0 \leq p \leq 1) .
$$

The parameter $\lambda_{1}$ controls the location $(\mu)$ of the GLD, $\lambda_{2}$ controls the scale $(\sigma)$, and $\lambda_{3}$ and $\lambda_{4}$ jointly determine the skew $\left(\alpha_{3}\right)$ and kurtosis $\left(\alpha_{4}\right)$ of the distribution. ${ }^{6}$ Substituting a value of $p$ into the expression above produces the value of $x=R(p)$, which corresponds to the $p^{\text {th }}$ percentile of the GLD. If $n$ values of $p$ are obtained from a uniform random number generator, the resulting values of $x$ represent a random sample from the distribution defined by the GLD.

By selecting appropriate values for $\lambda_{3}$ and $\lambda_{4}$, distributions having particular degrees of skew (positive to negative) and kurtosis (platykurtic to leptokurtic) can be approximated. Tables provided in Ramberg et al. (1979, pp. 210-214) present the values of $\lambda_{1}-\lambda_{4}$ for GLDs having $\mu=0, \sigma=1,-2.0 \leq \alpha_{3} \leq+2.0$, and $1.8 \leq$ $\alpha_{4} \leq 15.8$. Since the values of $\lambda_{1}-\lambda_{4}$ in these tables define "standardized" GLDs, the percentile function above transforms the uniform random numbers to standard scores, $z_{i}$, sampled from the GLD. These scores may be scaled to $X_{i}$ as follows: ${ }^{7}$

$$
X_{i}=\mu+z_{i} \sigma .
$$

To generate simulated data for Mihal and Barrett's (1976) study, we simply select GLDs with the desired skew and kurtosis, use the GLDs to convert uniform random numbers to standard scores, and then scale these scores on the basis of the means and standard deviations in Table 1 . After some trial and error, the GLDs shown in Figure 1 were found to provide reasonable approximations of the

Table 1

Sample Statistics Reported by Mihal and Barrett (1976, p. 231)

\begin{tabular}{lccccccccc}
\hline & \multicolumn{8}{c}{ Predictor and Criterion Variables } \\
\cline { 2 - 9 } & $\begin{array}{c}\text { Rod and } \\
\text { Frame }\end{array}$ & $\begin{array}{c}\text { Embedded } \\
\text { Figures }\end{array}$ & $\begin{array}{c}\text { Initial } \\
\text { RT }\end{array}$ & $\begin{array}{c}\text { Simple } \\
\text { RT }\end{array}$ & $\begin{array}{c}\text { Choice } \\
\text { RT }\end{array}$ & $\begin{array}{c}\text { Complex } \\
\text { RT }\end{array}$ & $\begin{array}{c}\text { Selective } \\
\text { Attention }\end{array}$ & $\begin{array}{c}\text { Number } \\
\text { Accidents }\end{array}$ \\
\hline$M$ & 4.04 & 85.19 & .23 & .40 & .52 & .81 & 38.23 & $1.03^{*}$ \\
$S D$ & 3.36 & 45.26 & .02 & .04 & .05 & .14 & 32.63 & $.90^{*}$ \\
\hline
\end{tabular}

Note $-N=75$. ${ }^{*}$ Estimated (see text). 

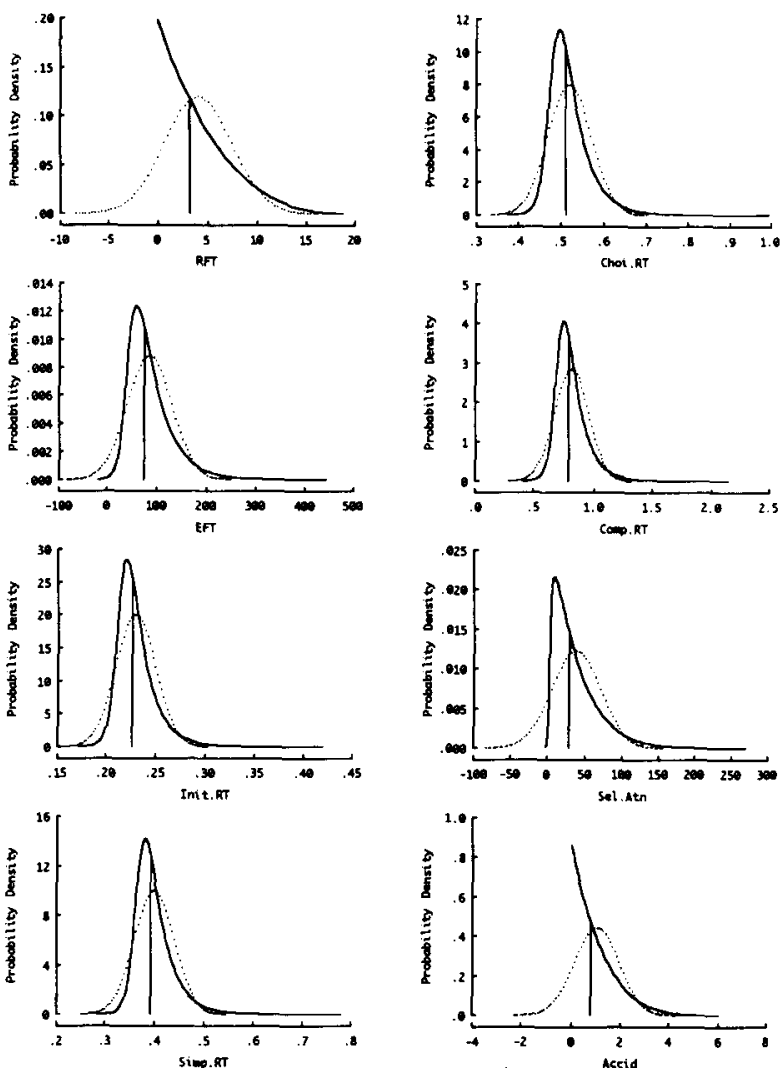

Figure 1. The GLDs used to approximate the variables from Mihal and Barrett's (1976) study.

(suspected) distributions of the variables in Mihal and Barrett's study. Both the RFT and accident variables required distributions with a mode near zero in order to accommodate the fact that the mean was so close to the lower scale limit. To a lesser extent, the same was true for the selective attention variable. All of the RT measures employed the same GLD, selected to approximate the usual positive skew associated with these data.

Table 2 summarizes the parameters used to approximate the variables of Mihal and Barrett's (1976) study. If we wish to achieve a very high degree of accuracy in simulating a study, the raw data for the study should be obtained, and sample estimates of $\alpha_{3}$ and $\alpha_{4}$ should be computed for each variable. These values would then be used to obtain GLDs from the tables in Ramberg et al. (1979) which have the same skew and kurtosis as the sample data. In the absence of the raw data, however, fairly reasonable distributions can be identified simply through trial and error. Using DATASIM, one can easily define and plot various GLDs. The probability density functions in Figure 1 , for example, were obtained by defining and then plotting each of the GLDs listed in Table 2. (This is done by using the LAMBDA and PDF commands.)

Now, suppose we use a uniform random number generator (Lewis \& Orav, 1989, p. 65) to generate $k=8$ columns of $N=75$ values of $p$, where $0 \leq p \leq 1$. Next, transform the $p_{i}$ in each column to $z_{i}$ by using the values of $\lambda_{1}-\lambda_{4}$ in Table 2. Finally, scale the $z_{i}$ to $X_{i}$ by using the values of $\mu$ and $\sigma$ in the table, and round the values as appropriate. The resulting distributions represent random samples from the populations defined in Table 2 . The means, standard deviations, and distribution shapes willin the long run-be the same as those specified for the populations (Figure 1). However, since the uniform random numbers generated for the eight columns are uncorrelated, so are the resulting columns of $X$ values. Obviously, we want the simulated data to show the same correlation structure as reported in Mihal and Barrett's (1976) study. Some way must therefore be found to introduce the desired intercorrelation among the eight variables.

Table 3 presents the actual correlations among the predictor and criterion variables reported by Mihal and Barrett (1976, p. 232). Allowing for sampling error, we want our simulated data sets to reproduce the correlation structure shown in this table. Graybill describes a procedure for factoring a $k \times k$ correlation matrix, $\mathbf{R}$, into an upper and lower triangular matrix, $\mathbf{T}$ and $\mathbf{T}^{\prime}$, such that $\mathbf{R}=\mathbf{T}^{\prime} \mathbf{T}$ (Graybill, 1969, p. 298). The interesting thing about $\mathbf{T}$ is that if we generate an $N \times \mathbf{k}$ matrix $\mathbf{Z}$ of uncorrelated standard normal deviates, and compute $\mathbf{C}=\mathbf{Z T}$, the values in the $k$ columns of $\mathbf{C}$ will be correlated-in the

Table 2

Parameters Defining the Generalized Lambda Distributions (GLDs)

\begin{tabular}{ccccccccc}
\hline & \multicolumn{7}{c}{ Predictor and Criterion Variables } \\
\cline { 2 - 9 } Parameters* & $\begin{array}{c}\text { Rod and } \\
\text { Frame }\end{array}$ & $\begin{array}{c}\text { Embedded } \\
\text { Figures }\end{array}$ & $\begin{array}{c}\text { Initial } \\
\text { RT }\end{array}$ & $\begin{array}{c}\text { Simple } \\
\text { RT }\end{array}$ & $\begin{array}{c}\text { Choice } \\
\text { RT }\end{array}$ & $\begin{array}{c}\text { Complex } \\
\text { RT }\end{array}$ & $\begin{array}{c}\text { Selective } \\
\text { Attention }\end{array}$ & $\begin{array}{c}\text { Number } \\
\text { Accidents }\end{array}$ \\
\hline$\mu$ & 4.04 & 85.19 & .23 & .40 & .52 & .81 & 38.23 & 1.03 \\
$\sigma$ & 3.36 & 45.26 & .02 & .04 & .05 & .14 & 32.63 & .90 \\
$\alpha_{3}$ & 1.10 & 1.60 & 1.80 & 1.80 & 1.80 & 1.80 & 1.70 & 1.40 \\
$\alpha_{4}$ & 3.80 & 7.60 & 10.60 & 10.60 & 10.60 & 10.60 & 7.00 & 5.00 \\
$\lambda_{1}$ & -1.215 & -.693 & -.532 & -.532 & -.532 & -.532 & -1.001 & -1.132 \\
$\lambda_{2}$ & .1582 & -.0258 & -.1349 & -.1349 & -.1349 & -.1349 & .0412 & .1092 \\
$\lambda_{3}$ & .0000 & -.004383 & -.0299 & -.0299 & -.0299 & -.0299 & .001027 & .0000 \\
$\lambda_{4}$ & .2379 & -.0218 & -.0931 & -.0931 & -.0931 & -.0931 & .0441 & .1411 \\
\hline${ }_{\mu}=E(X), \sigma$ & $=\sqrt{E}(\bar{X}-\mu)^{2}, \alpha_{3}=E(X-\mu)^{3} / \sigma^{3}, \alpha_{4}=E(X-\mu)^{4} / \sigma^{4}$. & &
\end{tabular}


Table 3

Correlation Matrix Reported by Mihal and Barrett (1976, p. 232)

\begin{tabular}{lccccccc}
\hline \multicolumn{1}{c}{ Predictor } & $\begin{array}{c}\text { Embedded } \\
\text { Figures }\end{array}$ & $\begin{array}{c}\text { Initial } \\
\text { RT }\end{array}$ & $\begin{array}{c}\text { Simple } \\
\text { RT }\end{array}$ & $\begin{array}{c}\text { Choice } \\
\text { RT }\end{array}$ & $\begin{array}{c}\text { Complex } \\
\text { RT }\end{array}$ & $\begin{array}{c}\text { Selective } \\
\text { Attention }\end{array}$ & $\begin{array}{c}\text { Number } \\
\text { Accidents }\end{array}$ \\
\hline Rod and frame & $.53 \ddagger$ & -.22 & -.07 & .02 & $.26 *$ & $.46 \ddagger$ & $.38 \ddagger$ \\
Embedded figures & & -.18 & -.05 & .11 & $.23^{*}$ & $.44 \ddagger$ & $.24^{*}$ \\
Initial RT & & & $.65 \ddagger$ & $.33 \dagger$ & $.23^{*}$ & -.10 & -.11 \\
Simple RT & & & & $.37 \dagger$ & $.23^{*}$ & .00 & .15 \\
Choice RT & & & & & $.65 \ddagger$ & $.45 \ddagger$ & .15 \\
Complex RT & & & & & & $.44 \ddagger$ & $.27 \dagger$ \\
Selective attention & & & & & & & $.40 \ddagger$ \\
\hline
\end{tabular}

Note $-N=75 . \quad{ }^{*} p<.05 . \quad \dagger p<.01 . \quad \ddagger p<.001$.

Table 4

Factor Solution, $R=T^{\prime} T$, for the Correlation Matrix Reported by Mihal and Barrett (1976)

$\mathbf{T}^{\prime}=\left[\begin{array}{rrrrrrrr}1.0000 & .0000 & .0000 & .0000 & .0000 & .0000 & .0000 & .0000 \\ .5300 & .8480 & .0000 & .0000 & .0000 & .0000 & .0000 & .0000 \\ -.2200 & -.0748 & .9726 & .0000 & .0000 & .0000 & .0000 & .0000 \\ -.0700 & -.0152 & .6513 & .7554 & .0000 & .0000 & .0000 & .0000 \\ .0200 & .1172 & .3528 & .1898 & .9085 & .0000 & .0000 & .0000 \\ .2600 & .1087 & .3036 & .0690 & .5634 & .7115 & .0000 & .0000 \\ .4600 & .2314 & .0190 & .0309 & .4415 & .0542 & .7319 & .0000 \\ .3800 & .0455 & -.0236 & .2551 & .1068 & .1345 & .2088 & .8455\end{array}\right]$

long run-as specified by $\mathbf{R}$ (Bradley, 1989b, p. 169). That is,

$$
\underset{k \times k}{\mathbf{R}}=\underset{k \times k}{\mathbf{T}^{\prime}} \underset{k \times k}{\mathbf{T}}, \quad \underset{N \times k}{\mathbf{C}}=\underset{N \times k}{\mathbf{Z} \times k \times k} \underset{k}{\mathbf{T}} .
$$

The factor solution for Mihal and Barrett's correlation matrix is presented in Table 4 . To generate correlated data by using the matrix $\mathrm{T}$, we simply generate an $N \times k$ matrix of uniform random numbers, convert the uniforms to a matrix $\mathbf{Z}$ of standard normal deviates (Box \& Muller, 1958 ), and then multiply $\mathbf{T}$ by $\mathbf{Z}$. If we want normal distributions for our variables, all we have to do is scale the standard scores in each column to $X$, and round. However, if we want nonnormal distributions, the correlated standard scores must be transformed back to uniform numbers (percentiles), the uniforms transformed using the appropriate GLD, and the resulting (nonnormal and correlated) $z$ scores scaled and rounded as necessary. In doing this, we make one concession: the long-run expected values for the correlations among the variables will be somewhat lower than those specified by the matrix $\mathbf{R}$. This is because Graybill's factoring procedure is strictly applicable only to multinormal distributions. ${ }^{8}$ The magnitude of the bias introduced by using nonnormal distributions is examined below (see Table 6).

\section{Initializing a Simulation}

The techniques reviewed above are used by DATASIM to generate multivariate data sets (Bradley, 1989b, pp. 168-171). The first step in simulating a study is to initialize the simulation. This consists of specifying the research design, the number of observations to be generated, and the values of any population parameters (e.g., $\mu, \sigma, \varrho)$ needed to control the simulation. Nonnormal population shapes, labels for the variables, upper and lower limits for data values, and the number of decimal places to retain in the simulated data may also be specified. All of this is done by entering appropriate commands or by executing a command macro. After the simulation 
is initialized, any number of simulated data sets can be generated, analyzed, and plotted.

To illustrate, consider the initialization of Mihal and Barrett's (1976) study shown in Figure 2. ${ }^{9}$ The functions of the various commands, such as MU, SIGMA, RHO, and LAMBDA, are self-evident. The parameter values entered after these commands are taken from Tables 2 and 3. Once the simulation is initialized, the command SEED will generate, and DATA will generate and display a simulated data set. Each time SEED or DATA is entered, DATASIM initializes the random number generator with a randomly selected seed, outputs the value of this seed for reference, and then generates an $N \times k$ data set. If the SEED command is supplied with a user-specified value (e.g., SEED 29384561), DATASIM will generate a data set based on that value rather than one selected at random. Consequently, any data set that has ever been generated for a particular initialization can be regenerated by simply entering SEED followed by the appropriate value. ${ }^{10}$ Once a data set has been generated, the data can be plotted, analyzed, and displayed (PLOT, HIST, STAT, CORR, REGR, DISPLAY, etc.).

To generate and display a simulated data set for Mihal and Barrett's (1976) study, the initialization commands in Figure 2 would be entered first, and then the command DATA. As it turns out, Mihal and Barrett's study is one of a large number of studies included in the DATASIM library. This means that the initialization commands, as well as a complete description of the study, have already been saved in appropriate subdirectories of the library. Consequently, there is no need to initialize the simulation directly, as in Figure 2. Instead, the user can ask DATASIM to describe the study, initialize the simulation, and generate a simulated data set. Figure 3 shows how to do this for the built-in problem ACCIDENT, which was designed to simulate Mihal and Barrett's study. Entering the command HOMEWORK VIEW ACCIDENT (or selecting Other $\rightarrow$ Problem $\rightarrow$ View from the menu bar) opens a view window containing a complete description of the study as well as a simulated data set. ${ }^{11}$ By scrolling through the window, the user can conveniently review both the description and data: Figure 4, for example, shows the vertical scroll button positioned to display the end of the description and the beginning of the simulated data. Note that a complete reference to the original study (Mihal \& Barrett, 1976) is provided so that students can consult the original source for additional information. Also, the seed used to generate the simulated data set is displayed. This seed is important, because it allows the student or instructor to regenerate this specific data set at a later time, if desired.

After reviewing the description and data, the user can click on the command window in order to analyze and plot the data. Figure 5 shows the sample statistics and intercorrelations obtained for the simulated data. Allowing for sampling error, the means and standard deviations are in reasonably good agreement with the population values specified in the initialization. Also, the correlations in the last column of the matrix replicate, for the most part, the findings of Mihal and Barrett (1976). As in the original study, the RFT, complex RT, and selective attention measures correlate significantly with accident rates $(r=.457$, $r=.326$, and $r=.641$, respectively, $p<.01$ ). In contrast with the results of the original study, the EFT measure does not correlate significantly with accidents $(r=$

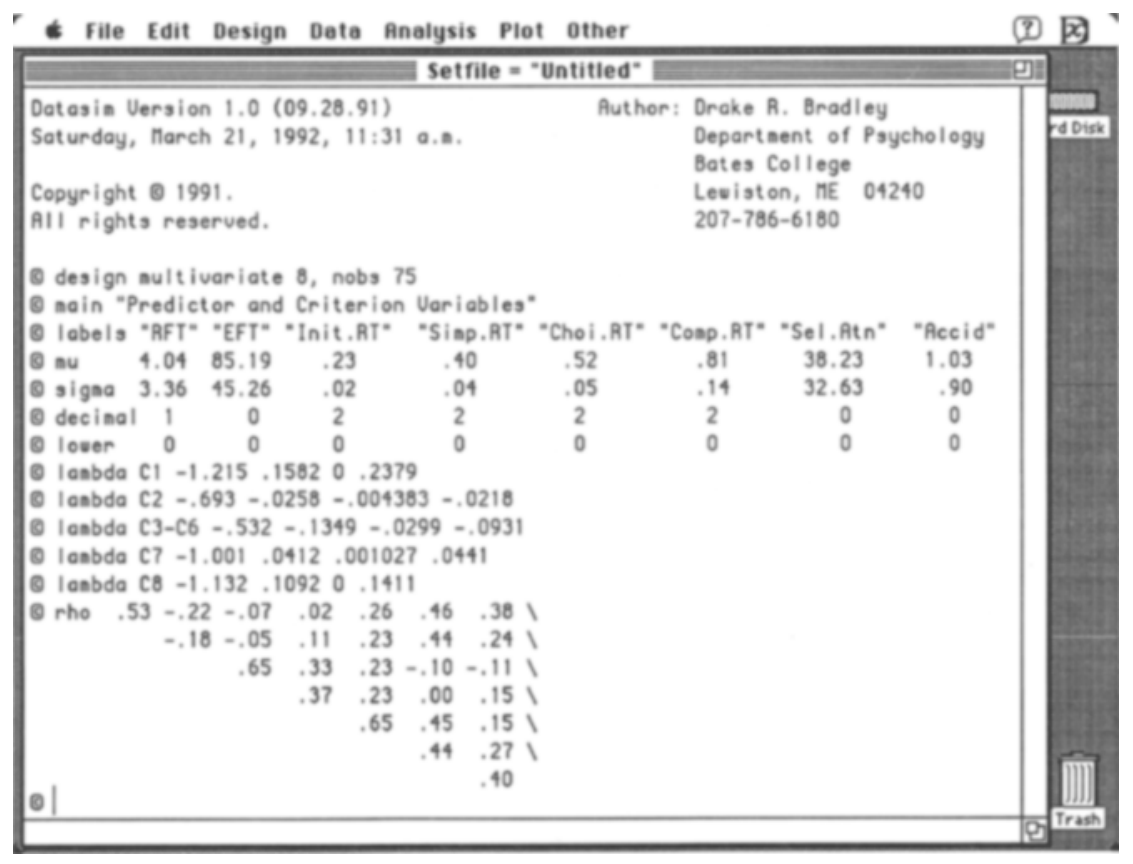

Figure 2. Initializing a DATASIM simulation of Mihal and Barrett's (1976) study. 


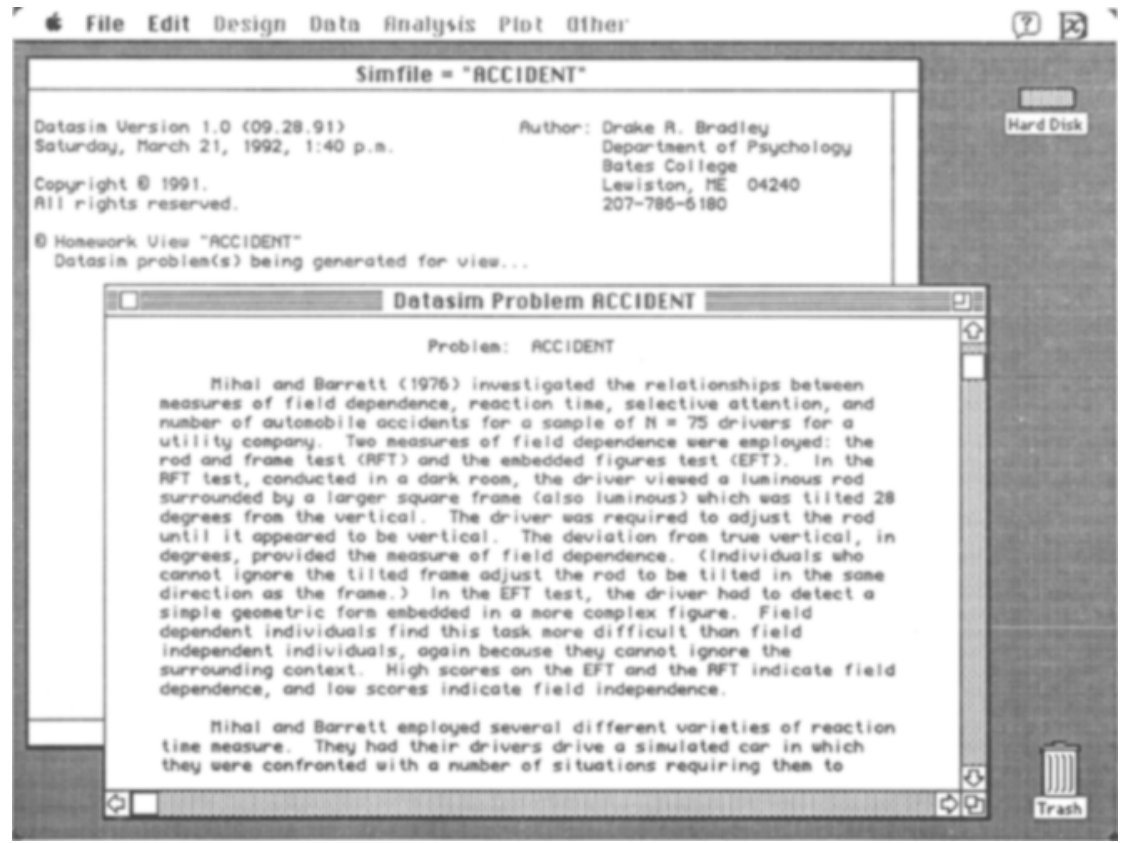

Figure 3. Viewing a description of the DATASIM problem ACCIDENT.

$.151)$, whereas the choice RT measure does $(r=.436)$. Such variation in outcomes occurs because of sampling error in the correlations. Figure 6 displays the histograms for each of the variables in the simulated data set. Comparison of these distributions with the theoretical distributions in Figure 1 shows that the simulated data are following the GLDs specified in the initialization. Given the nonnormal distributions, we would not expect scatterplots of the relationships between pairs of variables to have the usual oval shape associated with bivariate normal distributions. Figure 7 reveals that this is indeed the case: the scatterplot of EFT versus RFT shows the fanning-out effect associated with positively skewed distributions. Furthermore, several of the scatterplots suggest that the re-

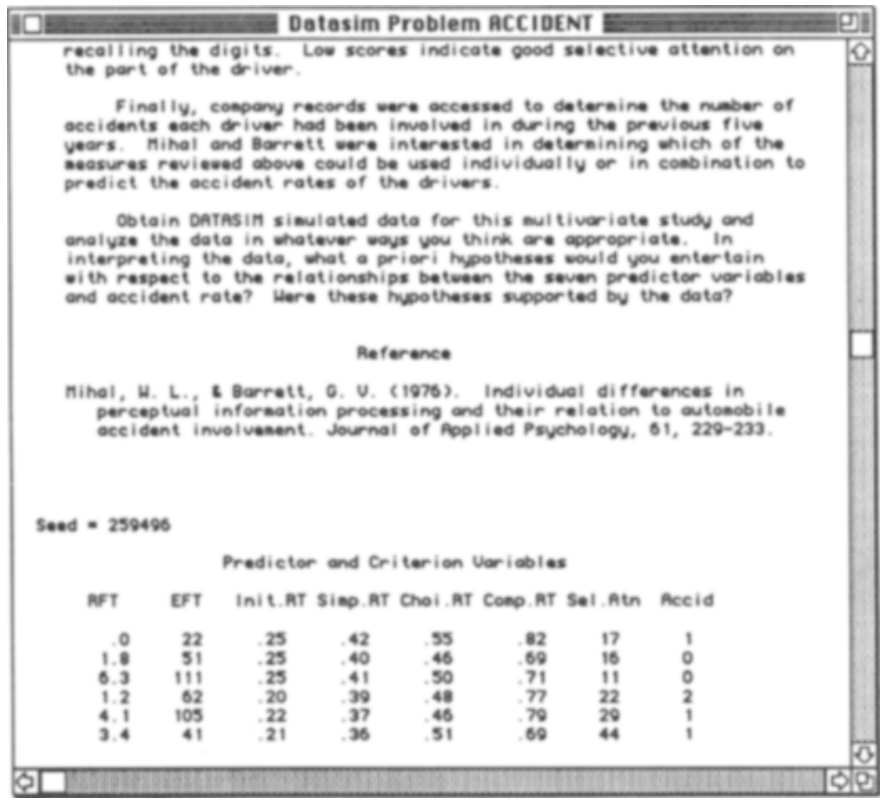

Figure 4. Viewing the simulated data for the DATASIM problem ACCIDENT. 


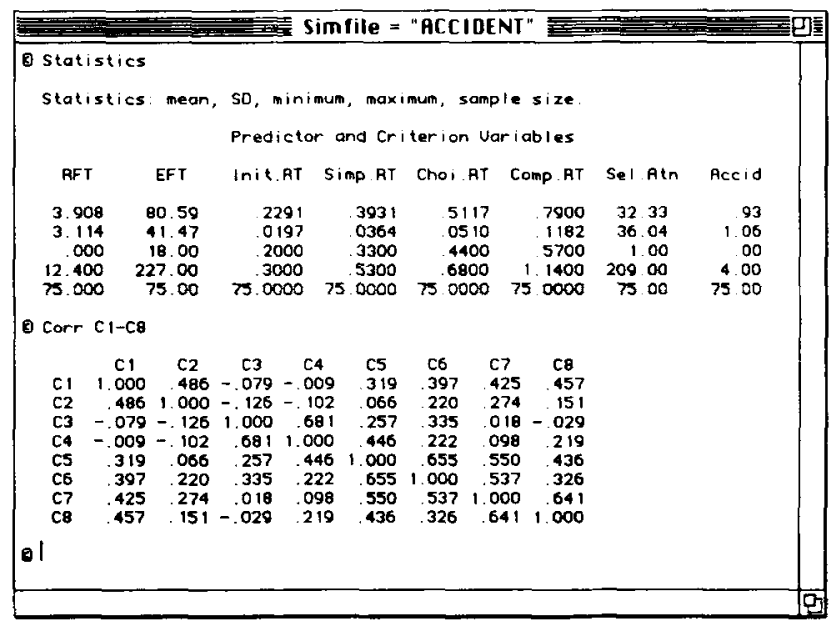

Figure 5. Sample statistics and intercorrelations for the simulated data.

sidual variance in $Y$ is not constant across the values of $X$. The presence of nonnormality and heteroscedasticity in the data might explain why Mihal and Barrett did not report a multiple linear regression analysis on the data.

Despite the violations of the normality and homoscedasticity assumptions, it is of some interest to examine the regression solution for the simulated data. Figure 8 shows the results of regressing the accident variable against the
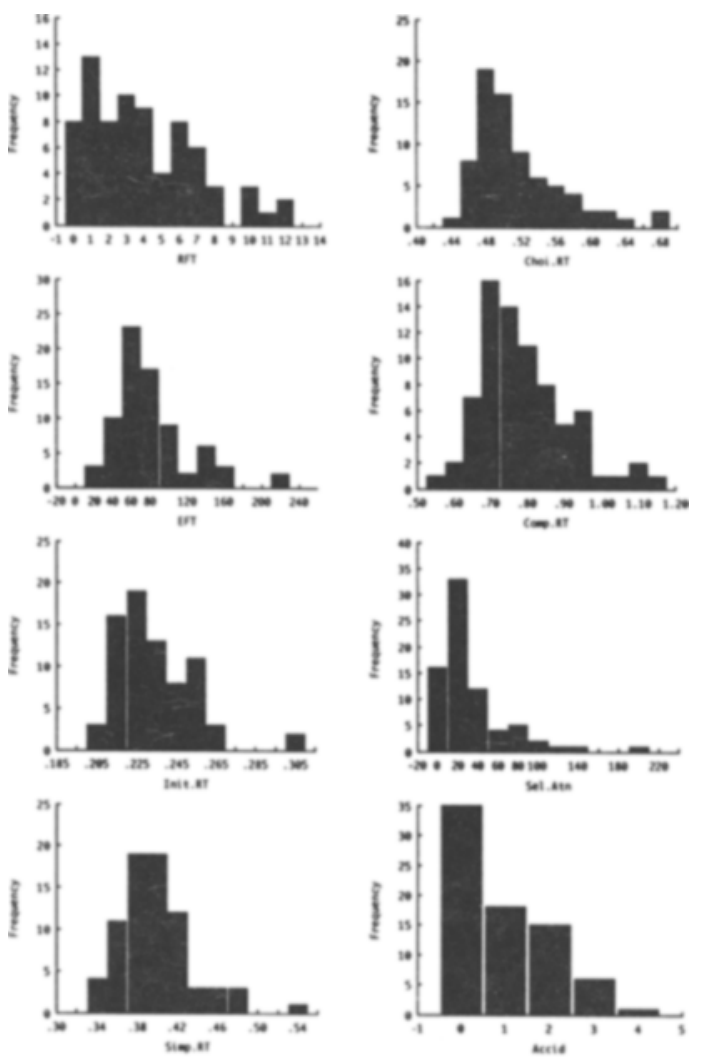

Figure 6. Histograms of the simulated data for the eight variables. seven predictors. It can be a very useful exercise to have students compare the $t$ tests on the regression coefficients to the corresponding tests on the sample $r$ s. For example, even though the choice and complex RT measures are significantly correlated with accidents, the corresponding partial slopes for these variables do not differ significantly from zero. This occurs because the choice and complex RT measures share substantial amounts of variance with the RFT, EFT, and selective attention measures, and when this is taken into account, they do not account for unique portions of variance in the dependent variable (accidents). Conversely, although the initial and simple RT measures do not correlate significantly with accidents $(r=-.029$ and $r=.219$, respectively), the partial slopes for these variables differ significantly from zero (or nearly so: $p=$ .0645 and $p=.0136$, respectively). Since the initial and simple RT measures do not share much variance with the RFT, EFT, and selective attention measures, their otherwise weak correlations with accidents are free to contribute in full to the regression solution. Suppressor effects are also apparent in the data: although the RFT and EFT measures both correlate positively with accidents, the partial slope for RFT is positive and that for EFT is negative. Since RFT and EFT are positively correlated, the negative slope for the EFT measure in the multiple regression solution appears to be suppressing "unwanted" variation in the predicted variable associated with the RFT. In any event, a careful examination of the correlation and regression coefficients obtained for simulated data sets can help students to grasp the relationship between the two better, as well as reveal a number of interesting regression phenomena.

\section{Large- $\boldsymbol{N}$ Simulations of Mihal and Barrett}

Although the simulated data generated for the problem ACCIDENT are in general agreement with the data reported by Mihal and Barrett (1976), the sampling error in the various statistics makes it difficult to evaluate the quality of the simulation. For testing purposes, we can temporarily increase the sample size to some arbitrarily large value, 

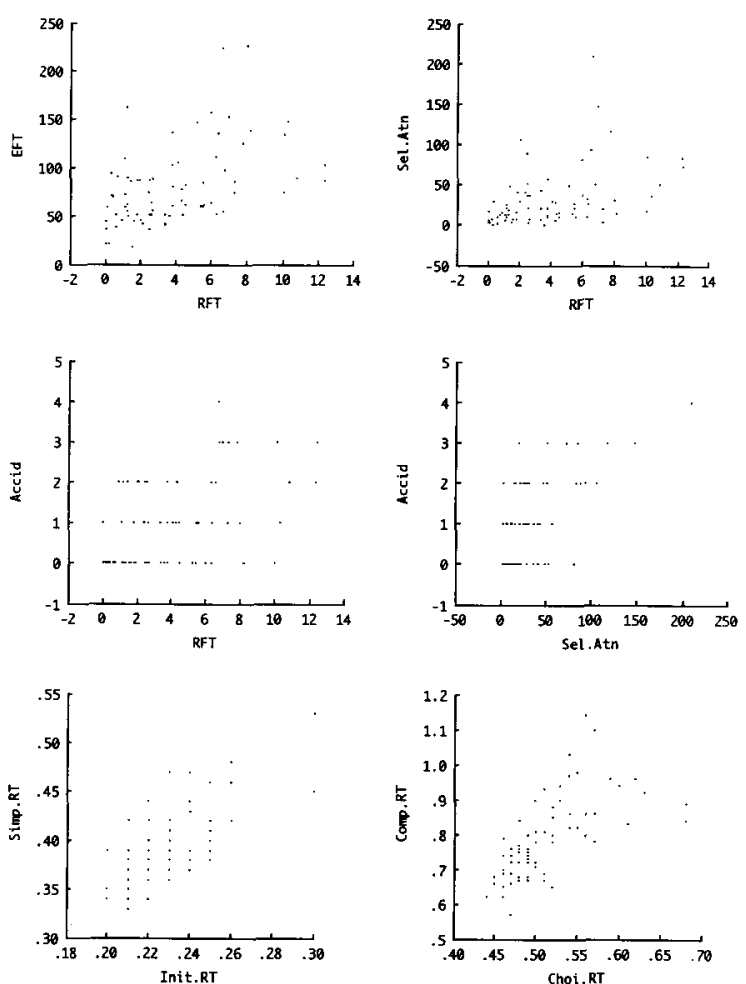

Figure 7. Scatterplots of selected pairs of variables from the simulated data. generate a new simulated data set, and reanalyze the data. The reduction in sampling error would then permit a more precise comparison to be made between the sample and population values.

We can anticipate that certain inaccuracies will arise in the simulated data. As noted above, using nonnormal distributions will attenuate the correlations among the variables to some extent. Moreover, the use of a continuous distribution (GLD) to model a discrete distribution (accidents) will introduce some bias in the value of the mean and standard deviation. This occurs because rounding continuous values to integers "loses" some of the information in the data. Finally, imposing a lower scale limit of $X=0$ for each variable can produce bias. Although the GLD selected to approximate each variable was chosen to minimize the generation of off-scale values, there is a small but finite probability that negative values will arise. When the LOWER command is specified, as in Figure 2, such off-scale values are assigned the value of the lower limit. Other things being equal, this will shift the mean up and the standard deviation down a bit, relative to the values specified for $\mu$ and $\sigma$.

The first large- $N$ simulation, Simulation 1 , assessed the effects of nonnormality, rounded values, and scale limits on biasing the sample statistics. The same initialization as in Figure 2 was employed, except that the sample size, NOBS, was set to $N=70,000$ rather than $N=75 .^{12}$ A simulated data set was generated by entering SEED

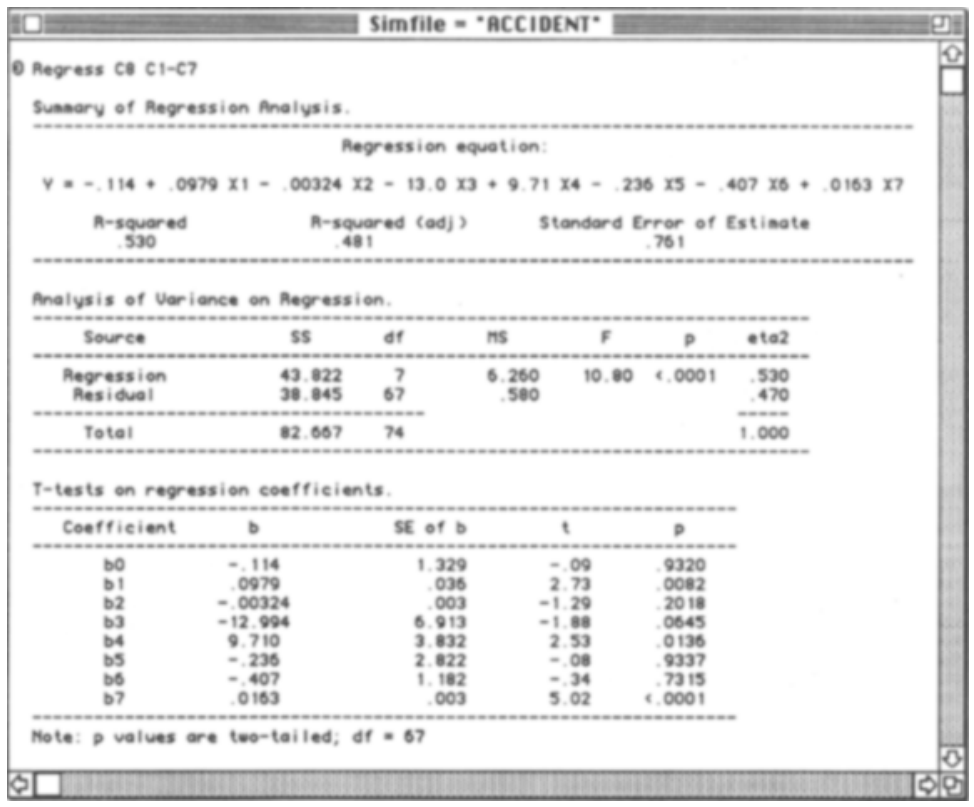

Figure 8. A multiple regression analysis of the simulated data. 
Table 5

Population versus Sample Statistics for Simulations 1 and $2(N=70,000)$

\begin{tabular}{cccccccccc}
\hline & \multicolumn{7}{c}{ Predictor and Criterion Variables } \\
\cline { 2 - 10 } Statistic & $\begin{array}{c}\text { Rod and } \\
\text { Frame }\end{array}$ & $\begin{array}{c}\text { Embedded } \\
\text { Figures }\end{array}$ & $\begin{array}{c}\text { Initial } \\
\text { RT }\end{array}$ & $\begin{array}{c}\text { Simple } \\
\text { RT }\end{array}$ & $\begin{array}{c}\text { Choice } \\
\text { RT }\end{array}$ & $\begin{array}{c}\text { Complex } \\
\text { RT }\end{array}$ & $\begin{array}{c}\text { Selective } \\
\text { Attention }\end{array}$ & $\begin{array}{c}\text { Number } \\
\text { Accidents }\end{array}$ \\
\hline$M(\mu)$ & & & & & & & & \\
Pop. & 4.04 & 85.19 & .23 & .40 & .52 & .81 & 38.23 & 1.03 \\
Sim 1 & 4.050 & 84.93 & .2300 & .4000 & .5200 & .8099 & 38.11 & $.99 \dagger$ \\
Sim 2 & 4.051 & 84.93 & .2300 & .4000 & .5200 & .8097 & 38.11 & 1.03 \\
SD (o) & & & & & & & & \\
Pop. & 3.36 & 45.26 & .02 & .04 & .05 & .14 & 32.63 & .90 \\
Sim 1 & 3.369 & $44.99 *$ & $.0202 \dagger$ & .0398 & .0500 & .1404 & 32.62 & $.98 \dagger$ \\
Sim 2 & 3.362 & 45.19 & .0200 & .0400 & .0500 & .1404 & 32.64 & .90 \\
Minimum & & & & & & & & \\
Sim 1 & 0.000 & 0.00 & .1600 & .2400 & .3300 & .3200 & 0.00 & 0.00 \\
Sim 2 & -8.984 & -92.01 & .1530 & .2348 & .3173 & .2541 & -89.76 & -2.51 \\
Maximum & & & & & & & & \\
Sim 1 & 19.600 & 566.00 & .4800 & .9300 & 1.2900 & 3.0500 & 337.00 & 7.00 \\
Sim 2 & 17.166 & 267.54 & .3083 & .5583 & .7250 & 1.3893 & 171.95 & 4.81 \\
\hline Not
\end{tabular}

Note-Simulation 1 employed nonnormal distributions, rounded $X$ values, and lower limits of $X=0$; Simulation 2 employed normal distributions, unrounded values, and no scale limits. Onesample $z$ test comparing sample with population value: ${ }^{*} p<.05, \dagger p<.001$.

168752265 , and sample means, standard deviations, intercorrelations, and multiple regression statistics were computed. A second large- $N$ simulation, Simulation 2, eliminated the potential biases caused by nonnormal distributions, rounding errors, and the imposition of scale limits. The initialization was identical to that of Simulation 1 with the following exceptions. First, a normal distribution was specified for all eight variables by entering LAMBDA C1-C8 0.1974 .1349 .1349 . These values of $\lambda_{1}-\lambda_{4}$ define a GLD with $\alpha_{3}=0$ and $\alpha_{4}=3$ (Ramberg et al., 1979 , p. 210). Second, the command DECIMAL 10 was entered to eliminate the rounding of data values (DATASIM does not round if $d \geq 10$ decimal places are specified). Finally, no lower limits were specified, to avoid reassigning negative values to $X=0$. A simulated data set with $N=70,000$ was generated by entering SEED 168752265 , and the sample statistics were computed. Note that the use of identical seeds for Simulations 1 and 2 means that the same $N \times k=560,000$ uniform random numbers $\left(p_{i j}\right)$ were generated for each simulation. The only difference, then, consists in how these numbers were scaled by the GLDs, and in whether or not the resulting numbers were rounded and subjected to scale limits. This strategy allows any differences in the sample statistics for the two simulated data sets to be attributed to these factors, rather than to differences in the particular uniform random numbers employed. Moreover, given the large sample size, very little sampling error is expected in the sample statistics, so precise comparisons with the population values are possible.

The results of the large- $N$ simulations are presented in Tables 5, 6, and 7. For comparison, each table includes the population parameters employed in the initialization. Table 5 presents the means, standard deviations, and minimum and maximum values obtained for the eight variables in Simulations 1 and 2, denoted $\operatorname{Sim} 1$ and $\operatorname{Sim} 2$.
To assess the accuracy of the simulations, one-sample $z$ tests were conducted to compare the sample and population values. ${ }^{13}$ For Simulation 2 , none of the means or standard deviations differed significantly from the population values. For Simulation 1, the mean number of accidents was significantly less than the population value, .99 versus $1.03(z=-10.99, p<.001)$. The standard deviations of the EFT, initial RT, and accident variables in Simulation 1 also differed significantly from the corresponding population values. Although the latter differences are due in part to the effects of rounding and/or scale limits, the lack of robustness to nonnormality of the

Table 6

Population versus Sample $r$ s for Simulations 1 and $2(N=\mathbf{7 0 , 0 0 0})$

\begin{tabular}{cccccccr}
\hline \multicolumn{7}{c}{ Intercorrelations } \\
\hline$r$ & Pop. & Sim 1 & Sim 2 & $r$ & Pop. & Sim 1 & Sim 2 \\
\hline$r_{12}$ & .530 & $.502 \ddagger$ & .530 & $r_{45}$ & .370 & $.340 \ddagger$ & .371 \\
$r_{13}$ & -.220 & $-.188 \ddagger$ & -.217 & $r_{48}$ & .230 & $.207 \ddagger$ & .229 \\
$r_{14}$ & -.070 & $-.063 *$ & -.070 & $r_{47}$ & .000 & .001 & -.001 \\
$r_{15}$ & .020 & .019 & .020 & $r_{48}$ & .150 & $.126 \ddagger$ & .148 \\
$r_{16}$ & .260 & $.244 \ddagger$ & .262 & & & & \\
$r_{17}$ & .460 & $.425 \ddagger$ & .459 & $r_{56}$ & .650 & $.624 \ddagger$ & .653 \\
$r_{18}$ & .380 & $.342 \ddagger$ & .377 & $r_{57}$ & .450 & $.417 \ddagger$ & .448 \\
& & & & $r_{58}$ & .150 & $.128 \ddagger$ & .146 \\
$r_{23}$ & -.180 & $-.156 \ddagger$ & -.180 & & & & \\
$r_{24}$ & -.050 & -.044 & -.050 & $r_{67}$ & .440 & $.412 \ddagger$ & .444 \\
$r_{25}$ & .110 & $.099 \dagger$ & .107 & $r_{68}$ & .270 & $.235 \ddagger$ & .268 \\
$r_{26}$ & .230 & $.209 \ddagger$ & .229 & & & & \\
$r_{27}$ & .440 & $.413 \ddagger$ & .442 & $r_{78}$ & .400 & $.353 \ddagger$ & .399
\end{tabular}

$r_{28} \quad .240 \quad .215 \ddagger \quad .241$

$r_{34} \quad .650 \quad .616 \neq \quad .651$

$\begin{array}{llll}r_{35} & .330 & .300 \neq & .333\end{array}$

$\begin{array}{llll}r_{36} & .230 & .208 \ddagger & .233\end{array}$

$\begin{array}{llll}r_{37} & -.100 & -.084 \ddagger & -.098\end{array}$

$\begin{array}{llll}r_{38} & -.110 & -.095 \ddagger & -.112\end{array}$

Note-One-sample $r$-to- $z$ test comparing sample $r$ with population $Q$ : ${ }^{*} p<.05, \dagger p<.01, \ddagger p<.001$. 
Table 7

Population versus Sample Regression Statistics for Simulations 1 and $2(N=70,000)$

\begin{tabular}{|c|c|c|c|c|c|c|c|c|c|c|}
\hline \multirow[b]{2}{*}{ Case } & \multicolumn{10}{|c|}{ Regression Statistics } \\
\hline & $\beta_{0}$ & $\beta_{1}$ & $\beta_{2}$ & $\beta_{3}$ & $\beta_{4}$ & $\beta_{3}$ & $\beta_{6}$ & $\beta$, & $R^{2}$ & $\sigma_{\text {res }}$ \\
\hline Pop. & .564 & .0529 & -.000908 & -12.0 & 7.70 & -2.25 & 1.08 & .00787 & .285 & .761 \\
\hline Sim 1 & $.339^{*}$ & $.0576^{*}$ & $-.000544 *$ & $-10.0^{*}$ & $6.53 *$ & $-1.70^{*}$ & $.94 *$ & $.00725^{*}$ & $.221^{*}$ & $.862 *$ \\
\hline $\operatorname{Sim} 2$ & .628 & .0517 & -.000898 & -12.1 & 7.76 & -2.35 & 1.08 & .00793 & .284 & .761 \\
\hline
\end{tabular}

Note-Tests comparing sample with population value: ${ }^{*} p<.001$.

$z$ test for a standard deviation may have produced spurious significance in the tests. However, the difference between the Simulation 1 standard deviation for accidents and the population value, $S=.98$ versus $\sigma=.90$, was probably caused by rounding the continuous values of the GLD to integers. Given the shape of the GLD used to approximate the accident distribution (Figure 1), it looks as if rounding values in the interval $0<X<1$ would move more values down to " 0 " than up to " 1 ." The greater cumulative error associated with such "downward" rounding would decrease the sample mean, just as is observed in Simulation 1. If one assumes that the upper tail of the accident distribution remains relatively fixed, this shift would also cause an increase in the standard deviation.

The minimum and maximum values obtained for Simulations 1 and 2 reflect the distribution shapes, rounding effects, and scale limits used to generate the data for each variable. In contrast with the values from Simulation 1 , which never generates negative values, the minimum values for the RFT, EFT, selective attention, and accident variables in Simulation 2 are considerably less than zero. This reflects the absence of a scale limit and the fact that the left tails of the normal distributions for these variables extend further into the negative numbers than the left tails of the GLDs (see Figure 1). Conversely, the larger maximum values obtained in Simulation 1 are due to the positive skew of the GLDs. These effects are apparent in Figure 9, which plots the frequency distribution of the RFT variable for Simulations 1 (top) and 2 (bottom). The large number of observations in these histograms allows the underlying theoretical distributions to be clearly discerned.

Table 6 presents the intercorrelations obtained for the two large- $N$ simulations. As expected, the sample correlations for Simulation 1 are consistently smaller than the corresponding population values. For Simulation 2 , on the other hand, the sample and population values are in excellent agreement. Fisher's $r$-to- $z$ transformation was used to compare each of the sample $r$ s to the corresponding population es. The nonnormal distributions of Simulation 1 significantly attenuate all but three of the sample $r s$. Although the differences are not large in an absolute sense, they are consistent: .530 versus .502 for $r_{12},-.220$ versus - 188 for $r_{13}$, and so on, with the largest discrepancy being .400 versus .353 for $r_{78}$. In contrast, none of the sample correlations in Simulation 2 differ significantly from the population values, with the largest dis- crepancy being .440 versus .444 for $r_{67}$. Table 7 presents the population regression parameters and the corresponding sample regression statistics for Simulations 1 and 2 . The sample regression statistics were obtained by entering REGRESS $\mathrm{C} 8 \mathrm{Cl}-\mathrm{C} 7$, and the population regression parameters were obtained by entering REGINFO $\mathrm{C} 8 \mathrm{Cl}-\mathrm{C} 7$. One-sample $t$ tests were computed to compare each of the sample $b_{i}$ s to the population $\beta_{i}$. For Simulation 1 , all the sample regression coefficients differed significantly from the population values $(p<.001)$; for Simulation 2 , on the other hand, none did so. Likewise, tests comparing the values of $R^{2}$ and $S_{\text {res }}$ to the population were significant for Simulation 1, but not Simulation 2 .

What do the results of these large- $N$ simulations imply about the accuracy of DATASIM as a multivariate data generator? The Simulation 1 results reveal that systematic biases may arise when one attempts to generate
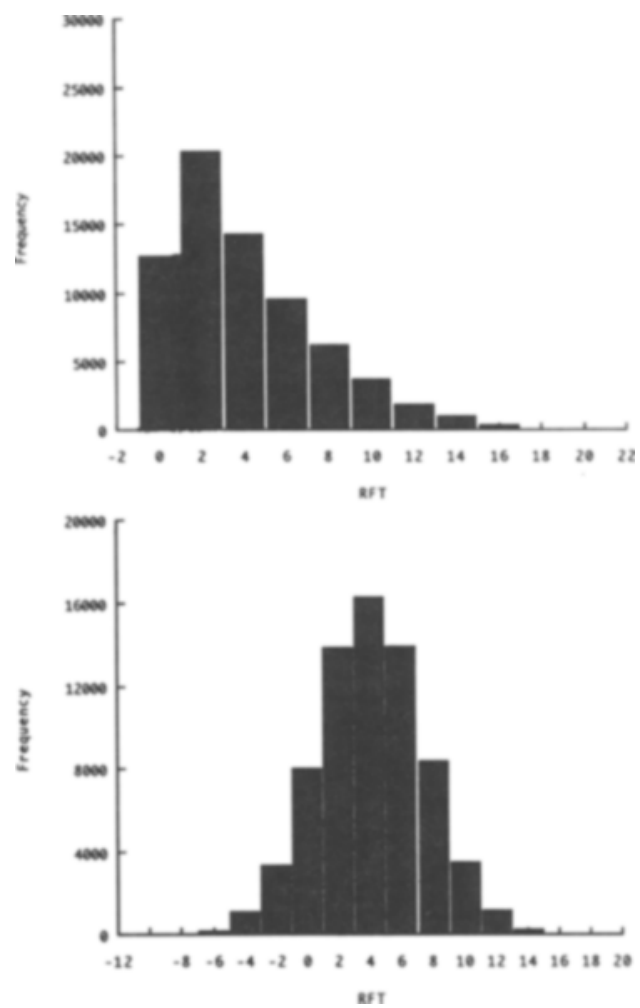

Figure 9. Histograms of the rod-and-frame test data for Simulations 1 (nonnormal) and 2 (normal). 
"realistic" data sets having nonnormal distributions, discrete values, and on-range data. The biases occur because the data-generating algorithms assume a continuous multinormal distribution without truncation (i.e., scale limits). If we attempt to model data sets that depart from these assumptions, the results will necessarily be approximate rather than exact. Tables 5-7 show that the magnitude of the bias is not large, and for the purpose of generating data sets for homework assignments and in-class demonstrations, the level of accuracy provided by Simulation 1 is probably sufficient. Higher levels of accuracy can be achieved with additional effort, however. For example, the CATVAR command could be used to initialize the accident measure as a categorical variable (Bradley, 1988, p. 66). This command allows the user to specify the probability of generating each of several discrete values of $X$. This would remove the rounding bias produced by converting GLD values to integers. Also, the attenuation in the correlations caused by nonnormality can, in some instances, be offset by initializing a simulation with values of $\varrho$ that are somewhat larger than those actually desired. As a first approximation, the size of the adjustments should be proportional to the amount of attenuation observed in the $r$ for a large- $N$ simulation using the original (unadjusted) values. In Table 6, for instance, the difference between the population and Simulation 1 correlations could be used as an initial estimate of the increase required in the adjusted population values. A large- $N$ simulation using the adjusted values could then be conducted, and the discrepancies between the actual and desired correlations could be used to estimate a new set of adjustments. A few iterations should suffice in most cases to obtain an adjusted correlation matrix that compensates for the attenuation caused by the nonnormal distributions.

Of course, Simulation 2 conforms to the model requirements of the data generator, and the results in Tables 5, 6 , and 7 show that the resulting sample statistics are in very close agreement with the population values. Given the extremely large sample size employed $(N=70,000)$, this simulation provides a highly sensitive test of the accuracy of DATASIM's algorithms for generating multivariate data sets. And although one cannot "prove" the null hypothesis, it seems safe to say that if biases are present in the data-generating algorithms, they must be of a very small magnitude to escape detection by Simulation 2 . In conjunction with previous research (Bradley, 1991; Bradley et al., 1990), this suggests that DATASIM is functioning properly as a general-purpose data simulator.

\section{Large-Scale Sampling Experiments of Mihal and Barrett}

The preceding sections demonstrated the generation of single simulated data sets for Mihal and Barrett's (1976) study, using both small- and large- $N$ samples. However, a unique feature of DATASIM is its ability to generate and analyze large numbers of simulated data sets. The results of any statistical analyses computed on the data sets can be stored in a file and subsequently processed in various ways. For example, counts of the number of Type I or Type II decision errors can be tabulated and displayed. Or, the empirical sampling distribution of a particular statistic $\left(z, t, F, r, \chi^{2}\right)$ can be compared with a corresponding theoretical distribution by plotting the cumulative distribution functions and performing a KolmogorovSmirnov analysis. Finally, the various statistics saved in the file can be read into DATASIM as raw data, and plotted and analyzed by using the full complement of plotting and analysis routines. These capabilities allow students and researchers to conduct large-scale sampling experiments to investigate the effects of violating assumptions on the outcomes of statistical tests. In fact, as part of a systematic program of research undertaken to test the datagenerating algorithms of DATASIM, a number of previous analytical and empirical studies have been successfully replicated (Boneau, 1960; Box, 1954; Bradley, Bradley, McGrath, \& Cutcomb, 1979; Bradley \& Cutcomb, 1977; Collier, Baker, Mandeville, \& Hayes, 1967; Hsu, 1938; Hsu \& Feldt, 1969; Norton, 1952; Scheffé, 1959). It is perhaps ironic that in conducting these tests we have uncovered errors in some of the "exact" Type I error rates

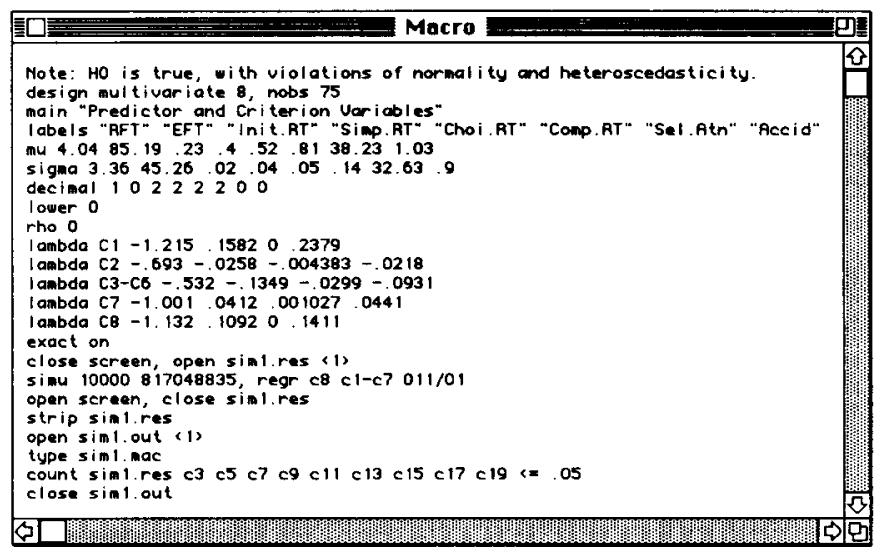

Figure 10. Command macro for conducting a DATASIM sampling experiment. 
calculated by Box (1954) and Hsu (1938), as well as in some of the empirical Type I error rates reported by Norton (1952) and Collier et al. (1967). ${ }^{14}$

In this final section, we illustrate how to conduct a DATASIM sampling experiment. Specifically, we investigate the effects of the nonnormal and heteroscedastistic distributions characterizing the Mihal and Barrett study on the Type I error rates of the tests conducted in a multiple regression analysis. Figure 10 shows a command macro designed to do this. The macro consists of three parts. The first part initializes the simulation: the parameters specified (MU, SIGMA, RHO, etc.) are identical to those shown in Figure 2, except that the values of $e_{i j}$ are set to zero for all $i$ and $j$. Doing this assures that the null hypotheses for the overall regression $\left(H_{0}: R^{2}=0\right)$ and the individual partial slopes $\left(H_{0}: \beta_{i}=0\right)$ are true. Tests of the corresponding hypotheses on the simulated data sets will therefore produce Type I errors whenever $p \leq \alpha$. The second part of the command macro performs the sampling experiment. This involves generating a large number of simulated data sets, conducting a multiple regression analysis on each, and directing the results to a file. The command SIMULATE is used to perform repetitive simulation in DATASIM: in the present case, a total of 10,000 data sets are generated from a "master" seed of 817048835 ; and an analysis of variance on the regression of $\mathrm{C} 8$ against $\mathrm{C} 1-\mathrm{C} 7$, as well as $t$ tests on the individual regression coefficients, are computed for each. The results of these analyses are directed to an output file, SIM1.RES. Finally, the third part of the macro processes the results of the sampling experiment. This involves stripping the output file of text, so that only $F$ ratios, $t$ ratios, and $p$ levels remain, and then counting the number of times $p \leq \alpha=.05$ for each of the tests. The resulting counts of Type I errors, as well as a listing of the command macro itself, are saved in another output file (SIM1.OUT) for archival purposes. ${ }^{15}$

Executing the macro in Figure 10 will allow us to ascertain whether the rather substantial violations of the normality and homoscedasticity assumptions that (probably) characterized Mihal and Barrett's (1976) data cause dramatic changes in the risk of making a Type I error. The results are shown in Table 8, which presents the actual proportion of Type I errors obtained for the $F$ test on $R^{2}$, and for each of the $t$ tests comparing a regression coefficient, $b_{i}$, to $\beta_{i}=0$. As is evident, the actual Type I error rates are quite close to the nominal level of $\alpha=.05$. (Note that the "error rate" for $\beta_{\mathrm{o}}$ is in fact the power of the corresponding $t$ test: given that $\varrho_{i s}=0$ for each of the seven predictor variables, $i=1-7$, the value of the $Y$ intercept would be $\beta_{0}=\mu_{Y}=1.03$, and not zero as stipulated by the null hypothesis.) These results suggest that Mihal and Barrett could safely conduct multiple linear regression analyses on their data, without worrying about unacceptable inflations in the Type I error rates of the tests. The actual Type I error rate for the test on $R^{2}$, for instance, is .0503 . Given the patently nonnormal distributions employed (Figure 1), as well as the heteroscedasticity in the data (Figure 7), this suggests that the $F$ test is remarkably robust to such violations.

Of course, we would feel somewhat more confident in drawing the preceding conclusion if we could compare the entire sampling distribution of $F$ ratios with a corresponding theoretical $F$ distribution. With $k=8$ variables and $N=75$ observations, the $F$ test for the overall regression $\left(F=M S_{\text {reg }} / M S_{\text {res }}\right)$ has $k-1=7$ and $N-k=$ $67 d f$ for the numerator and denominator mean squares, respectively. The Kolmogorov-Smirnov test may be employed to compare the empirical sampling distribution of $F$ ratios in the file SIM1.RES to a theoretical $F$ distribution with $\nu_{1}=7$ and $\nu_{2}=67 d f$. This is done by entering the command KS SIM1.RES C2 F 767. As shown in Figure 11 , the cumulative distributions functions (CDFs) of the empirical and theoretical values of $F$ are virtually indistinguishable, with a maximum absolute vertical deviation of only $D=.0051(p>.20)$. Consequently, whatever effects nonnormality and heteroscedasticity are having on the sampling distribution of $F$, they are not detectable by this analysis. Given the large number of $F$ ratios $(10,000)$ making up the empirical CDF, the failure to detect a significant difference cannot be ascribed to inadequate power in the Kolmogorov-Smirnov test. And while we need to explore several other kinds of nonnormal multivariate populations, including one's having mixtures of positively and negatively skewed distributions, these preliminary findings lend reassurance to those wishing to employ regression techniques on data sets that clearly violate the distributional assumptions of the analysis.

Two additional sampling experiments were conducted to see whether the power levels of the tests would be substantially altered by violations. The first sampling experiment was initialized precisely as in Figure 2-that is, with nonnormal distributions, rounded values, scale limits, and nonzero values of $\varrho_{i j}$. A total of 10,000 simulated data sets were generated from a master seed of 817048835 , and the number of correct rejections of $H_{0}$ was tabulated for each of the tests. The empirical power levels are shown in the first row of Table 9 (Experiment 1 ). To determine whether the violations are affecting the power of the tests,

Table 8

Empirical Type I Error Rates for Simulations of Mihal and Barrett's (1976) Study

\begin{tabular}{|c|c|c|c|c|c|c|c|c|c|}
\hline \multirow[b]{2}{*}{ Error Rate } & \multicolumn{9}{|c|}{ Tests on $R^{2}$ and the Partial Slopes } \\
\hline & $R^{2}$ & $\beta_{0}$ & $\beta_{1}$ & $\beta_{2}$ & $\beta_{3}$ & $\beta_{4}$ & $\beta_{5}$ & $\beta_{6}$ & $\beta_{7}$ \\
\hline Type I & .0503 & .0668 & .0491 & .0500 & .0466 & .0499 & .0499 & .0516 & .0492 \\
\hline
\end{tabular}




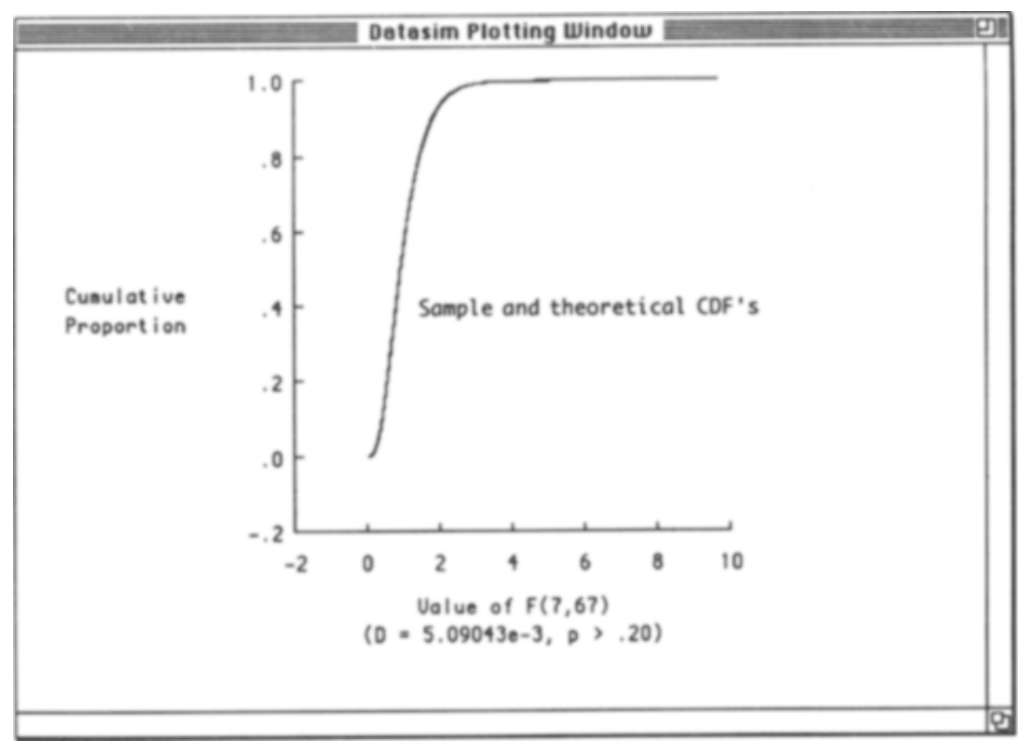

Figure 11. Sample and theoretical cumulative distribution functions for the $F$ test on the multiple regression solution.

we need to obtain an appropriate baseline. This requires initializing a simulation that employs normal distributions, unrounded values, and no scale limits. In addition, some adjustment is necessary in the intercorrelations specified, because the nonnormality employed in the first sampling experiment attenuates the correlations among the variables. The "Sim 1" correlations reported for the large$N$ simulation in Table 6 can be used to estimate the true population correlations that result from the nonnormal distributions. In order to have an "apples and apples"' comparison, therefore, the second sampling experiment employed the (reduced) Sim 1 correlations of Table 6 for the values of $\varrho_{i j}$ in the initialization. As before, a total of 10,000 simulated data sets were generated from a master seed of 817048835 , and the number of correct rejections of $H_{0}$ was tabulated for each of the tests. The results are shown in the second row of Table 9 (Experiment 2). Comparison of the power levels obtained in the two sampling experiments shows that violations of the distributional assumptions had only a minor impact on the power of the tests. As with the Type I error results, the violations that characterized Mihal and Barrett's (1976) data are unlikely to have substantially altered their likelihood of correctly rejecting $H_{0}$.

\section{Summary}

The software package DATASIM has been illustrated by simulating a multivariate study reported by Mihal and Barrett (1976). Only a relatively small number of commands are needed to initialize the simulation, and single or multiple data sets can be generated following the initialization. However, since Mihal and Barrett's study is one of 40 problems in the built-in library provided with the software, simulated data sets can be generated, plotted, and analyzed for this problem by simply initializing the appropriate command file (ACCIDENT). Large- $N$ simulations show that nonnormal distributions, scale limits, and discrete data introduce detectable but not large biases in the multivariate data sets for Mihal and Barrett's study. With normal distributions, unrounded data, and no scale limits, however, large- $N$ simulations show that DATASIMsimulated data sets have precisely the distributional properties and multivariate structure specified by the initialization. Finally, sampling experiments modeled on Mihal and Barrett's study show that the Type I error rates and power levels of the $F$ and $t$ statistics computed in a multiple linear regression analysis are close to the nominal levels, despite extreme violations of the normality and homoscedasticity assumptions.

Table 9

Empirical Power for Simulations of Mihal and Barrett's (1976) Study

\begin{tabular}{ccccccccccc}
\hline & \multicolumn{10}{c}{ Tests on $R^{2}$ and the Partial Slopes } \\
\cline { 2 - 11 } & $R^{2}$ & $\beta_{0}$ & $\beta_{1}$ & $\beta_{2}$ & $\beta_{3}$ & $\beta_{4}$ & $\beta_{3}$ & $\beta_{6}$ & $\beta_{7}$ \\
\hline Experiment 1 & .9001 & .0584 & .3143 & .0697 & .3057 & .4863 & .0984 & .1783 & .4211 \\
Experiment 2 & .8993 & .0582 & .3061 & .0542 & .3042 & .4674 & .0851 & .1490 & .4005 \\
\hline
\end{tabular}

Note-Rejections of $H_{0}: R^{2}=0$ and $H_{0}: \beta_{i}=0$ based on 10,000 simulated data sets. Sampling Experiment 1 had violations of assumptions; sampling Experiment 2 had no violations and employed adjusted $r$ (see text). 


\section{REFERENCES}

Boneau, C. A. (1960). The effects of violations of assumptions underlying the $t$ test. Psychological Bulletin, 57, 49-64.

Box, G. E. P. (1954). Some theorems on quadratic forms applied in the study of analysis of variance problems: I. Effect of inequality of variance in the one-way classification. Annals of Mathematical Staistics, 25, 290-302.

Box, G. E. P., MUlLER, M. E. (1958). A note on the generation of random normal deviates. Annals of Mathematical Statistics, 29, 610-611.

Bradley, D. R. (1988). DATASIM. Lewiston, ME: Desktop Press.

BRADLEY, D. R. (1989a). Computer simulation with DATASIM. Behavior Research Methods, Instruments, \& Computers, 21, 99-112.

BRADLEY, D. R. (1989b). A general purpose simulation program for statistics and research methods. In G. Garson \& S. Nagel (Eds.), $A d-$ vances in social science and computers (Vol. 1, pp. 145-186). Greenwich, CT: JAI Press.

BraDLEY, D. R. (1991a). Anatomy of a DATASIM simulation: The Doob and Gross horn-honking study. Behavior Research Methods, Instruments, \& Computers, 23, 190-207.

BradlEy, D. R. (1991b). DATASIM for the Macintosh. Lewiston, ME: Desktop Press

Bradley, D. R., Bradley, T. D., McGrath, S. G., \& Cutcomb, S. D. (1979). Type I error rate of the chi-square test of independence in $\mathrm{R} \times \mathrm{C}$ tables that have small expected frequencies. Psychological Bulletin, 86, 1290-1297.

Bradley, D. R., \& Cutcomb, S. (1977). Monte Carlo simulations and the chi-square test of independence. Behavior Research Methods \& Instrumentation, 9, 193-201.

Bradley, D. R., Hemstreet, R. L., \& Ziegenhagen, S. T. (1992), A simulation laboratory for statistics. Behavior Research Methods, Instruments, \& Computers, 24, 190-204.

Bradley, D. R., Senko, M. W., \& Stewart, F. A. (1990). Statistical simulation on microcomputers. Behavior Research Methods, Instruments, \& Computers, 22, 236-246.

Collier, R. O., Baker, F. B., Mandeville, G. K., \& Hayes, T. F. (1967). Estimates of test size for several test procedures based on conventional variance ratios in the repeated measures design. Psychometrika, 32, 339-353.

GRAYBILL, F. A. (1969). Introduction to matrices with applications in statistics. Belmont, CA: Wadsworth.

Hsu, P. L. (1938). Contribution to the theory of "Student's" $t$-test as applied to the problem of two samples. Statistical Research Memoirs, $2,1-24$

Hsu, T. C., \& FeLDT, L. S. (1969). The effect of limitations on the number of criterion score values on the significance level of the $F$ test. American Educational Research Journal, 6, 515-527.

LEWhs, P. A. W., \& Orav, E. J. (1989). Simulation methodology for statisticians, operations analysts, and engineers (Vol. 1). Pacific Grove, CA: Wadsworth.

Mihal, W. L., \& BARReTt, G. V. (1976). Individual differenences in perceptual information processing and their relation to automobile accident involvement. Journal of Applied Psychology, 61, 229-233.

MYкYTKA, E. F. (1978). Some useful properties and methods for determining the parameters of the Ramberg-Schmeiser-Tukey distribution. Unpublished masters thesis, University of Iowa.

Norton, D. W. (1952). An empirical investigation of some effects of non-normality and heterogeneity of the F-distribution. Unpublished doctoral dissertation, Iowa State University.

Ramberg, J. S., Dudewicz, E. J., Tadikamalla, P. R., \& Mykytka, E. F. (1979). A probability distribution and its uses in fitting data. Technometrics, 21, 201-214.

RAMBERG, J. S., \& SChMEISER, B. W. (1972). An approximate method for generating symmetric random variables. Communications of the $A C M, 15,987-990$.

RAMBerg, J. S., \& Schmeiser, B. W. (1974). An approximate method for generating asymmetric random variables. Communications of the $A C M, 17,78-82$.

SCHEFFÉ, H. (1959). The analysis of variance. New York: Wiley.
Witkin, H. A., Dyk, R. D., Faterson, H. F., Goodenough, D. R., \& KARP, S. A. (1962). Psychological differentiation: Studies of development. New York: Wiley.

\section{NOTES}

1. Datasim is available for IBM, IBM-compatible, and Macintosh computers. A minimum of $640 \mathrm{~K}$ of RAM is recommended for the IBM, and $760 \mathrm{~K}$ of RAM for the Macintosh.

2. DATASIM can make plots of main effect, simple effect, and interaction means, as well as scatterplots, histograms, polygons, bargraphs, and functions of the form $Y=f(X)$. The probability density functions used to generate simulated data can also be plotted. In addition to the usual battery of statistical procedures-one- and two-sample $z$ and $t$ tests, simple and multiple regression, part and partial correlation, one- and two-way classification $\chi^{2}$, and one- or two-sample tests on correlations, proportions, and variances-DATASIM also conveniently analyzes the data for one-way, two-way, and three-way factorial designs having any combination of independent- and correlated-group factors. In addition to the overall analysis of variance, DATASIM can compute tests of simple effects, simple-simple effects, simple interaction effects, pairwise and nonpairwise comparisons, and trend analyses. The latter two sets of analyses can be conducted at the main effect, simple effect, or simple-simple effects levels of the design matrix. Pooled error terms are automatically selected for the tests requiring them, and unweighted means analyses are conducted for designs having unequal $n$ on one or more of the independent-group factors. A complete set of nonparametric routines is planned for a furure release of DATASIM.

3. As will be shown later, the estimated value of $R^{2}$ for Mihal and Barrett's (1976) data (regressing accidents against all seven predictor variables) is $R^{2} \approx .285$ (Table 7 ). By contrast, the sum of the individual $r^{2}$ values for the seven predictors is .492 . The difference of $.492-$ $.285=.207$ is due to (shared) variance among the predictors that is redundant in predicting accidents.

4. By default, DATASIM generates data for a multivariate normal distribution. Consequently, distribution shapes need to be specified explicitly only when nonnormal distributions are desired. As shown later, this is done by the LAMBDA command.

5. A lower scale limit of $X=0$ would be only $z=(X-\mu) / \sigma=$ $(0-4.04) / 3.36=-1.20$ standard deviations below the mean. Since a normal distribution extends roughly \pm 3 standard deviations to either side of the mean, the RFT data cannot be normally distributed. And since the distribution is bounded by a lower but not an upper scale limit, this implies that the data are skewed to the right (positive skew).

6. The parameters $\alpha_{3}$ and $\alpha_{4}$ are the third and fourth standardized moments about the mean:

$$
\alpha_{3}=E(X-\mu)^{3 / \sigma^{3}} \quad \alpha_{4}=E(X-\mu)^{4} / \sigma^{4}
$$

7. If desired, the values of $\lambda_{1}$ and $\lambda_{2}$ from the tables can be adjusted so that the values of $x=R(p)$ can be generated directly from the GLD (Mykytka, 1978, p. 24):

$$
\lambda_{1}(\mu, \sigma)=\mu+\lambda_{1}(0,1) \sigma, \quad \lambda_{2}(\mu, \sigma)=\lambda_{2}(0,1) / \sigma .
$$

8. For example, the use of different distribution shapes for any two variables will introduce some degree of nonlinearity in the relationship between them. To illustrate, assume that two of the variables are perfectly correlated, $\varrho_{i j}=1.0$. In this case, the standard normal deviates in columns $i$ and $j$ of matrix $\mathbf{C}$ will be identical. However, when the $z$ scores are transformed back into uniform numbers, and then transformed to different GLDs, the resulting (nonnormal) $z$ scores will no longer be exactly equal, due to the differing amounts of "stretching" introduced by the GLDs. Hence the correlation between the nonnormal $z$ s will now be less than 1.0. A scatterplot of the values in column $i$ versus $j$ will show a nonlinear trend with no scatter off the curved line. Therefore, the product-moment correlations are reduced by differential nonnormality in the columns, but the rank-order correlations are not.

9. The screen dump shows DATASIM for the Macintosh (Version 1.0) running on a Macintosh Ilci. This version supports multiple windows, a menu-driven interface, and complete on-line documentation of com- 
mands. Note that commands may be either entered after the DATASIM command prompt, or selected by using the menu bar at the top of the screen.

10. This capability to seed the random number generator is a highly useful feature of DATASIM. It means that "customized" data sets illustrating interesting patterns and/or statistical issues can be easily created for demonstrations. Once a simulation is initialized, the instructor can enter SEED over and over again, plotting and analyzing the results for each data set, until one is found having precisely the right pattern or characteristics. The initialization commands and the current data sets's seed are then saved by entering SIMSAVE < filename $>$. At any later time, the instructor can enter EXECUTE < filename > and DATASIM will reinitialize the simulation and regenerate the simulated data set. Note that since the simulated data are not saved in the file-just the value of the seed-this makes for efficient use of disk space.

11. At present, the view window capability is available only on the Macintosh version of DATASIM. For the IBM version, the user would enter the commands DESCRIBE ACCIDENT, INITIALIZE ACCIDENT, and DATA in order to generate a description and simulated data set for Mihal and Barrett's (1976) study (the pause key is used to stop and resume scrolling of output on the screen). For both versions, DATASIM can save the problem description and data set on disk, and/or print them on a local printer. Finally, instructors can use the Book command to generate complete books of problem descriptions and individualized data sets for students to analyze and interpret throughout the semester. (BOOK problems are selected from the DATASIM library.)
12. A sample size of $N=70,000$ was the maximum that could be generated on a Macintosh IIci with 16 MB of RAM, and which allowed sufficient margin for computing the multiple regression solution. Running in 32-bit mode under System 7.0, a total of 14.5 MB of the available RAM were allocated to DATASIM.

13. The $z$ tests employed to compare the means and standard deviations with the corresponding population values were:

$$
z=\frac{\bar{X}-\mu}{\frac{\sigma}{\sqrt{N}}}, \quad z=\frac{S-\sigma}{\frac{\sigma}{\sqrt{2 N}}} .
$$

Although the $z$ test for the means is highly robust to nonnormality, given the central limit theorem and the very large $N(70,000)$, this is not the case with the $z$ test for the standard deviation. Consequently, the outcomes of the $z$ tests on the sample standard deviations for Simulation 1 (nonnormal distributions) cannot be entirely trusted.

14. Notes 6, 7, and 8 in a previous article (Bradley, Senko, \& Stewart, 1990) detail the errors discovered in Box (1954), Hsu (1938), and Norton (1954).

15. The sampling experiment conducted by the macro in Figure 10 requires fairly substantial computer resources. Even on a Macintosh IIci with a math coprocessor, the simulation will need approximately $12-16 \mathrm{~h}$ to finish. Substantial amounts of hard disk storage are also needed, because the unstripped results file, SIM1.RES, uses about $4.2 \mathrm{MB}$ of space (1.5 MB after stripping). 